'Escuela de Medicina, Facultad de Salud, Universidad Industrial de Santander (UIS). Bucaramanga, Colombia. ${ }^{2}$ Hospital Universitario de Santander. Bucaramanga, Colombia.

${ }^{3}$ Grupo Educativo para la Renovación de la Medicina Interna GERMINA. Bucaramanga, Colombia.

${ }^{4}$ Departamento de Medicina Interna. Bucaramanga, Colombia. ${ }^{5}$ Grupo de Investigación Paidós. Bucaramanga, Colombia. aEstudiante de Medicina. ${ }^{b}$ Médico Internista.

"Médico Internista, Oncólogo Clínico.

dMédico Pediatra, Magíster en Epidemiología.

Conflicto de Interés: Ninguno.

Recibido el 15 de mayo de 2018, aceptado el 23 de noviembre de 2018.

Correspondencia a: Juan Sebastián Arias Flórez, Calle 8 \#10-52, Convención, Colombia.

Tel: +573135840234 . juansebasar@hotmail.com

\section{Rendimiento diagnóstico de marcadores tumorales séricos convencionales en pacientes con sospecha clínica de cáncer primario metastásico a hígado}

\author{
JUAN SEBASTIÁN ARIAS-FLÓREZ $Z^{1,2,3, a}$, \\ ANGÉLICA MARÍA MARTÍNEZ-DELGADO ${ }^{1,2,3, a}$, \\ MARTHA LILIANA ALARCÓN-TARAZONA ${ }^{1,2,3,4, b}$, \\ JESÚS SOLIER INSUASTY-ENRIQUEZ ${ }^{1,2,3,4, \mathrm{c}}$, \\ LUIS ALFONSO DÍAZ-MARTÍNEZ ${ }^{1,5, \mathrm{~d}}$
}

\section{Conventional serum tumor markers in liver cancer. Retrospective analysis of 118 patients}

Background: Conventional serum tumor markers (CSTM) are widely used for monitoring patients with cancer. However, their usefulness as a diagnostic tool is controversial in primary or metastatic liver cancer (PMLC). Aim: To evaluate the diagnostic performance of the most commonly requested CSTM in the diagnostic approach of PMLC. Material and Methods: Review of medical records of patients aged over 18 years with a liver biopsy, attended from 2005 to 2017 in a tertiary hospital and a regional cancer center in Colombia. The results of liver biopsies were compared with tumor markers such as carcinoembryonic antigen (CEA), alpha-fetoprotein (AFP), CA 19-9, CA 125 and prostate specific antigen (PSA) using a receiver operating characteristic (ROC) curve analysis. Results: We reviewed 2063 medical records and retrieved 118 eligible patients (59 cases and 59 controls, 70\% males). Thirty percent had obstructive jaundice. There was heterogeneity in the amount of tumor markers requested according to medical criteria. Only CA 19-9 showed discriminative capacity ( $\geq 17.6 \mathrm{U} / \mathrm{m})$, with a cut-off point lower than that reported in the literature and a sensitivity of $69.5 \%$, specificity of $91.6 \%$, a positive likelihood ratio (LR) of 8.32, and a negative LR of 0.33. Conclusions: Except for CA 19-9, tumor markers were not useful for the initial diagnostic approach in patients with suspected primary or metastatic malignant liver tumors.

(Rev Med Chile 2018; 146: 1422-1428)

Key words: Biomarkers, Tumor; Liver Neoplasms; Mass Screening.
L os marcadores tumorales séricos convencionales (MTSC) se han convertido en los últimos años en una herramienta disponible en el abordaje de los pacientes con cáncer. Estos son sustancias bioquímicas producidas por las células tumorales o inducidas por el organismo en respuesta a su presencia, que en teoría reflejan su crecimiento, pueden permiten distinguir entre un proceso benigno o maligno y distinguir entre las diferentes neoplasias. Adicionalmente, después de confirmar el diagnóstico de cáncer, pueden ayudar a monitorizar el estado de la enfermedad durante y después del tratamiento ${ }^{1-3}$.

A pesar de su utilidad, el empleo de estas sustancias bioquímicas aún sigue siendo materia de discusión. El uso adecuado no sólo es crucial 
para el manejo de los pacientes con cáncer sino también para el correcto empleo de recursos y la disminución de pruebas invasivas en los pacientes. Los marcadores séricos no son específicos para malignidad ${ }^{1,3}$ ya que la mayoría de ellos son sintetizados también por células no tumorales; podria ser su concentración la que, en general, reflejaría la presencia del tumor ${ }^{4-6}$, puesto que se ha corroborado la existencia directa entre la carga tumoral y el nivel del marcador?

Un marcador tumoral ideal debe ser específico para detectar el tipo de cáncer y suficientemente sensible para descubrir pequeñas células tumorales; de esta manera sería posible hacer un diagnóstico temprano. Infortunadamente, de los MTSC conocidos, ninguno es suficientemente específico para un tumor en particular ni suficientemente sensible para un tamizaje; por esto las diferentes guías clínicas no lo recomiendan como método diagnostico salvo en muy contadas ocasiones ${ }^{4,8-10}$.

El uso de MTSC para el diagnóstico de cáncer continúa siendo debatible. Pocos estudios han evaluado la utilidad de los MTSC para este propósito en CPOMH, salvo el uso diagnóstico de la AFP en hepatocarcinoma. Con este trabajo se pretende evaluar el rendimiento diagnóstico de los MTSC más frecuentemente solicitados en pacientes con sospecha diagnóstica de CPOMH.

\section{Materiales y Métodos}

Se realizó un estudio retrolectivo de tecnología diagnóstica con muestreo trasversal. Se efectúo la revisión de historias clínicas en medio electrónico y físico de pacientes atendidos entre 2005 y 2017 en el Hospital Universitario de Santander y el Centro Oncológico Insuasty-Oncología de Bucaramanga, Colombia. Los criterios de inclusión eran ser $\geq 18$ años, sospecha de patología hepática presuntiva de cáncer primario o metastásico a hígado de novo diferentes a leucemias o mielomas, sin antecedente de cáncer o estado de embarazo. Todos los pacientes a incluir debían tener diagnóstico de certeza por estudio histopatológico de la lesión hepática.

Se definieron como caso los pacientes con inmunohistopatología positiva para cáncer y como controles aquellos en los que el resultado de la biopsia fuera negativo para cáncer, con o sin histopatología benigna. Se tuvieron en cuenta los MTSC tomados previo a la realización de la biopsia, incluyendo alfafetoproteina (AFP), antígeno carcinoembrionario (ACE), antígeno carbohidrato (CA) 19-9, CA 125 y el antígeno específico de la próstata (PSA), Los rangos de sus valores séricos se consideraron positivos para sospecha clínica de malignidad cuando los niveles séricos se encontraban según los valores reporatados en la literatura: ACE $>15 \mathrm{ng} / \mathrm{ml}$, AFP $>33 \mathrm{U} / \mathrm{ml}$, CA $19,9>200 \mathrm{U} / \mathrm{ml}$, CA $125>350 \mathrm{U} / \mathrm{ml}$ y PSA $>30$ $\mathrm{ng} / \mathrm{ml}^{11}$. La variable referencia o desenlace de oro fue el diagnóstico histopatológico de malignidad o benignidad, mientras que las predictoras fueron los MTSC. Adicionalmente, se consideraron variables independientes o de confusión los datos sociodemográficos y las variables clínicas relacionadas con los marcadores tumorales como la insuficiencia renal y hepática, prostatitis aguda, enfermedad hepática, ictericia, derrame pleural $\mathrm{y}$ ascitis.

Se realizó un análisis descriptivo de las características de cada grupo en porcentajes e intervalo de confianza de 95\% (IC 95\%); las variables continuas se describieron con medidas de tendencia central y de dispersión según sus características en promedio y desviación estándar (DE), o mediana y el rango intercuartil (RIQ). Para determinar si los valores de estas variables entre los dos grupos de pacientes eran estadísticamente significativos se realizó la prueba indicada según la naturaleza de las mismas o su distribución, considerando significativa cualquier diferencia con alfa $<0,05$.

De acuerdo con el comportamiento de los marcadores serológicos entre casos y controles se realizó análisis de la curva ROC para determinar si cada marcador tenía capacidad discriminativa; se calcularon indicadores de rendimiento diagnóstico y la concordancia más allá del azar de cada uno de los marcadores tumorales, teniendo especial cuidado en revisar tales indicadores de acuerdo con los puntos de corte descritos previamente. Los datos fueron analizados por medio de Stata 14.0 (StataCorp LC, College Station, Texas, Estados Unidos de América, 2015). El trabajo contó con el aval del comité de ética de cada la institución y siguió los lineamientos éticos nacionales e internacionales.

\section{Resultados}

Se revisaron 2.073 historias clínicas que cumplían los criterios de inclusión y exclusión, identificándose 118 pacientes, distribuidos en 59 con 
malignidad hepática, a manera de casos, y 59 sin esta, a manera de controles (Figura 1). La descripción de sexo y edad al igual que las comorbilidades se encuentra en la Tabla 1. En ambos grupos predominó el sexo masculino, pero fue mayor entre los controles. La comorbilidad más frecuente fue la enfermedad hepática y ascitis para los controles y los casos, respectivamente, aunque la proporción de personas con enfermedad hepática fue mayor entre los controles. Las presentaciones clínicas más frecuentes fueron ictericia obstructiva, derrame pleural, ascitis e insuficiencia hepática. La cirrosis fue la enfermedad hepática encontrada con mayor frecuencia, seguida por hepatitis B y absceso hepático.

Hubo heterogeneidad en la cantidad de marcadores tumorales disponibles para cada paciente (Tabla 2). Se encontraron diferencias en los valores para cada marcador tumoral; sin embargo, solo se evidenció significancia estadística para el valor de CA 19-9, cuya mediana y RIQ fue mayor entre los casos. Ahora bien, teniendo en cuenta, pero al comparar con los valores tomados como referencia, se encontraron diferencias en la proporción de pacientes con cifras anormales para el ACE, AFP y CA 125.

\section{Población de Pacientes $\geq 18$ años del Hospital Universitario de Santander y el Centro Oncológico Insuasty Oncología}

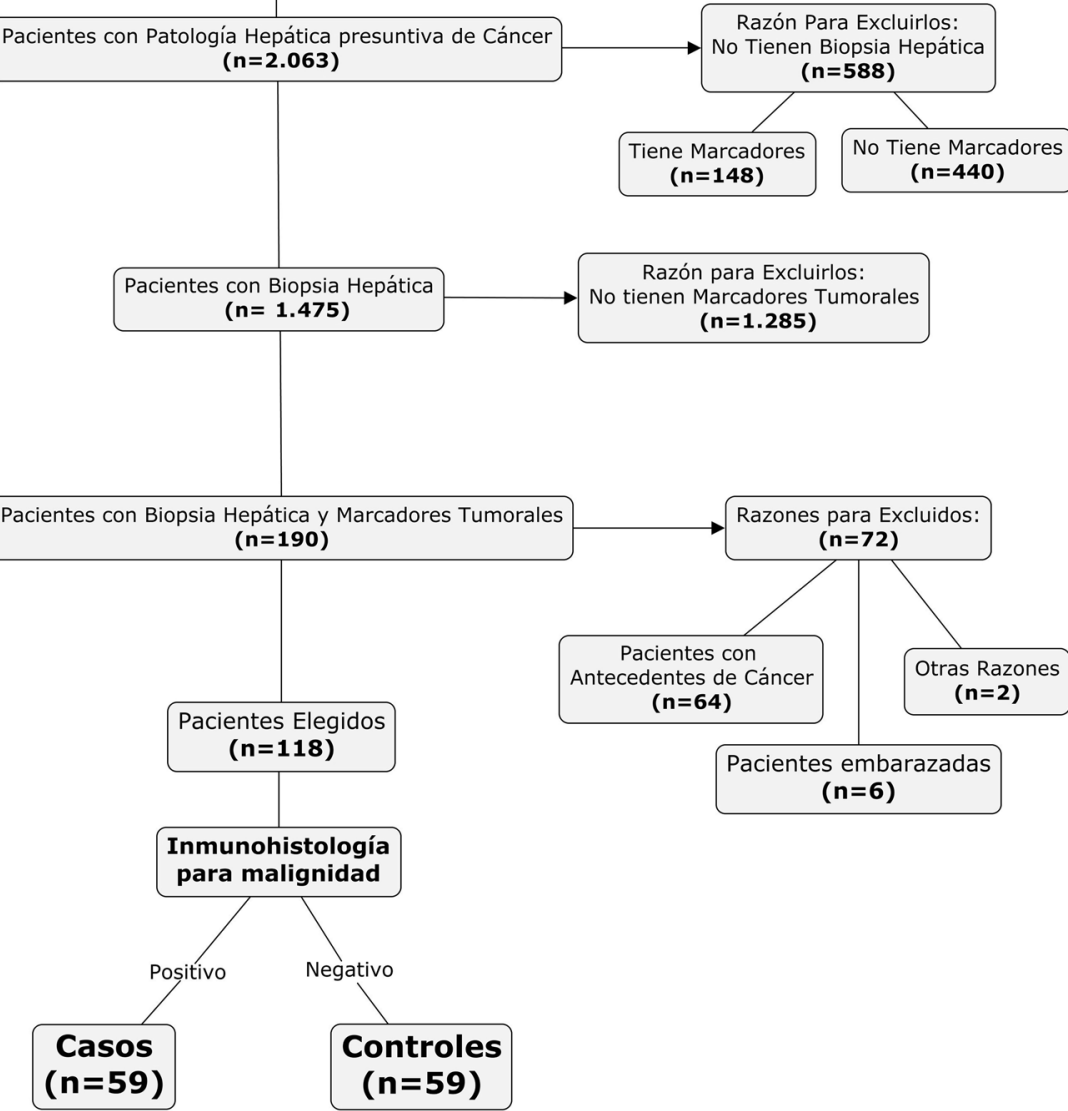

Figura 1. Flujograma de pacientes incluidos en el estudio. (Otras razones una muestra de biopsia correspondía a pulmón y otra el resultado histológico no era concluyente). 
La Tabla 3 describe los indicadores de rendimiento diagnóstico según el punto de corte predefinido, como la concordancia más allá del azar y, el área bajo la curva ROC exhibida en la Figura 2. Se confirma que tan solo el CA 19-9 tiene capacidad discriminativa para diferenciar entre posible malignidad y tumor benigno; el mejor punto de corte fue $\geq 17,6 \mathrm{U} / \mathrm{ml}$, a partir del cual se tiene sensibilidad de $69,5 \%$, especificidad del 91,6\% y razones de verosimilitud (LR) positiva de 8,32 y LR negativa de 0,33 (cuanto mayor es la desviación de la LR + de 1, más poderosa es una prueba positiva como predictor de un resultado positivo).

Tabla 1. Características de la población

\begin{tabular}{|lcccc|}
\hline Variable & Controles $(\mathbf{n}=\mathbf{5 9 )}$ & Casos $(\mathbf{n}=\mathbf{5 9})$ & Valor p & OR (IC95\%) \\
\hline Edad (años) & $54,5(16,2 \%)$ & $58,8(15 \%)$ & 0,132 & - \\
Sexo femenino & $13(20 \%)$ & $23(39 \%)$ & 0,046 & $2,26(0,94-5,55)$ \\
Comorbilidades & & & & \\
Insuficiencia renal & $7(20,3 \%)$ & $12(11,8 \%)$ & 0,210 & $0,52(0,16-1,60)$ \\
Insuficiencia hepática & $6(10,1 \%)$ & $6(10,1 \%)$ & 1,000 & $1,00(0,24-4,00)$ \\
Prostatitis aguda & $1(1,6 \%)$ & $0(0,0 \%)$ & 0,315 & Indeterminado \\
Enfermedad hepática & $22(37,2 \%)$ & $8(13,5 \%)$ & 0,003 & $0,26(0,09-0,70)$ \\
Ictericia obstructiva & $20(33,9 \%)$ & $15(25,4 \%)$ & 0,314 & $0,66(0,27-1,58)$ \\
Ictericia no obstructiva & $1(1,6 \%)$ & $0(0,0 \%)$ & 0,315 & Indeterminado \\
Derrame pleural & $19(32,2 \%)$ & $14(23,7 \%)$ & 0,305 & $0,65(0,26-1,58)$ \\
Ascitis & $17(28,8 \%)$ & $16(27,1 \%)$ & 0,837 & $0,91(0,37-2,21)$ \\
\hline
\end{tabular}

Tabla 2. Valores de los marcadores tumorales entre casos y controles

\begin{tabular}{|c|c|c|c|}
\hline Marcador & Controles & Casos & Valor $\mathbf{p}$ \\
\hline ACE & {$[n=17]$} & {$[n=49]$} & \\
\hline Mediana (RIQ) & $3,58(1,98$ a 5,25$)$ & $5,27(1,23$ a 16,13$)$ & 0,352 \\
\hline Valor anormal & $1(5,9 \%)$ & $14(28,6 \%)$ & 0,054 \\
\hline AFP & {$[n=35]$} & {$[n=45]$} & \\
\hline Mediana (RIQ) & $1,92(1,03$ a 6,41$)$ & $2,20(1,25$ a 22,49$)$ & 0,189 \\
\hline Valor anormal & $1(2,8 \%)$ & $11(24,4 \%)$ & 0,007 \\
\hline CA 19-9 & {$[n=12]$} & {$[n=23]$} & \\
\hline Mediana (RIQ) & $5,52(3,00$ a 10,88$)$ & $31,73(14,00$ a 500$)$ & $<0,001$ \\
\hline Valor anormal & $0(0,0 \%)$ & $9(39,13 \%)$ & 0,012 \\
\hline CA125 & {$[n=5]$} & {$[n=10]$} & \\
\hline Mediana (RIQ) & $41,15(9,50$ a 186,29$)$ & $144,12(64,43$ a 600$)$ & 0,176 \\
\hline Valor anormal & $2(40 \%)$ & $8(80 \%)$ & 0,121 \\
\hline PSA & {$[n=22]$} & {$[n=13]$} & \\
\hline Mediana (RIQ) & $1,42(0,68$ a 5,23$)$ & $1,50(0,70$ a 3,14$)$ & 0,851 \\
\hline Valor anormal & $0(0,0 \%)$ & $0(0,0 \%)$ & - \\
\hline
\end{tabular}

ACE: antígeno carcinoembrionario; AFP: alfafetoproteína; PSA: antígeno prostático específico. CA: Antígeno Carbohidrato. ACE y PSA son expresados en ng/ml; AFP. CA 19-9 y CA 125 expresado en U/ml. 
Tabla 3. Indicadores de rendimiento para ACE. AFP. CA 19-9. CA 125 y PSA (IC 95\%)

\begin{tabular}{|c|c|c|c|c|c|c|c|c|c|c|c|c|c|c|c|}
\hline & \multicolumn{3}{|c|}{ ACE > 15 ng/ml } & \multicolumn{3}{|c|}{ AFP > $33 \mathrm{U} / \mathrm{ml}$} & \multicolumn{3}{|c|}{ CA $19-9>200 \mathrm{U} / \mathrm{ml}$} & \multicolumn{3}{|c|}{ CA $125>350 \mathrm{U} / \mathrm{ml}$} & \multicolumn{3}{|c|}{ PSA > 30 ng/ml } \\
\hline & Casos & $\begin{array}{l}\text { Con- } \\
\text { troles }\end{array}$ & Total & Casos & $\begin{array}{l}\text { Con- } \\
\text { troles }\end{array}$ & Total & Casos & $\begin{array}{l}\text { Con- } \\
\text { troles }\end{array}$ & Total & Casos & $\begin{array}{l}\text { Con- } \\
\text { troles }\end{array}$ & Total & Casos & $\begin{array}{l}\text { Con- } \\
\text { troles }\end{array}$ & Total \\
\hline Positivo & 14 & 1 & 15 & 11 & 1 & 12 & 9 & 0 & 9 & 8 & 2 & 10 & 0 & 0 & 0 \\
\hline Negativo & 35 & 16 & 51 & 34 & 34 & 68 & 14 & 12 & 26 & 2 & 3 & 5 & 13 & 22 & 35 \\
\hline Total & 49 & 17 & 66 & 45 & 35 & 80 & 23 & 12 & 35 & 10 & 5 & 15 & 13 & 22 & 35 \\
\hline Sensibilidad & \multicolumn{3}{|c|}{$28,6 \%(14,9$ a 42,2$)$} & \multicolumn{3}{|c|}{$24,4 \%(10,8$ a 38,1$)$} & \multicolumn{3}{|c|}{$39,1 \%(17$ a 61,3$)$} & \multicolumn{3}{|c|}{$80 \%(50,2$ a 100$)$} & \multicolumn{3}{|c|}{$0,0 \%(0$ a 24,7$)$} \\
\hline Especificidad & \multicolumn{3}{|c|}{$94,1 \%(80$ a 100$)$} & \multicolumn{3}{|c|}{$97,1 \%(90,2$ a100) } & \multicolumn{3}{|c|}{$100 \%(95,8$ a 100$)$} & \multicolumn{3}{|c|}{$60 \%(7,1$ a 100$)$} & \multicolumn{3}{|c|}{$100 \%(97,7$ a 100$)$} \\
\hline VPP & \multicolumn{3}{|c|}{$99,3 \%(74,4$ a 100$)$} & \multicolumn{3}{|c|}{$91,7 \%$ (71,9 a 100) } & \multicolumn{3}{|c|}{$100 \%(94,4$ a 100$)$} & \multicolumn{3}{|c|}{$80 \%(50,2$ a 100$)$} & \multicolumn{3}{|c|}{ Indeterminado } \\
\hline VPN & \multicolumn{3}{|c|}{$31,4 \%(17,7$ a 45,1$)$} & \multicolumn{3}{|c|}{$50 \%(37,4$ a 62,6$)$} & \multicolumn{3}{|c|}{$46,2 \%(25,1$ a 67,2$)$} & \multicolumn{3}{|c|}{$60 \%(7,1$ a 100$)$} & \multicolumn{3}{|c|}{$62,9 \%(45,4$ a 80,3$)$} \\
\hline LR+ & \multicolumn{3}{|c|}{$4,86(0,69$ a 34,2$)$} & \multicolumn{3}{|c|}{$8,65(1,16$ a 63,15$)$} & \multicolumn{3}{|c|}{ Indeterminado } & \multicolumn{3}{|c|}{$2,0(0,65$ a 6,11$)$} & \multicolumn{3}{|c|}{ Indeterminado } \\
\hline LR- & \multicolumn{3}{|c|}{$0,76(0,61$ a 0,94$)$} & \multicolumn{3}{|c|}{$0,78(0,65$ a 0,93$)$} & \multicolumn{3}{|c|}{$0,61(0,44$ a 0,84$)$} & \multicolumn{3}{|c|}{$0,33(0,08$ a 1,39$)$} & Inde & etermina & ado \\
\hline Карра & 0,13 & $(0,02 \mathrm{a}$ & $0,25)$ & 0,18 & $(0,06 \mathrm{a}$ & $0,31)$ & 0,30 & $(0,10$ & $0,50)$ & 0,40 & $(0,09 a$ & $0,89)$ & Inde & etermina & \\
\hline aROC & 0,57 & $(0,43$ a & $0,71)$ & 0,58 & $(0,46$ a & $0,71)$ & 0,86 & $(0,75$ & $0,98)$ & 0,72 & $(0,43 a$ & $1,0)$ & 0,48 & $(0,28$ a & $0,68)$ \\
\hline
\end{tabular}

VPP: Valor predictivo positivo. VPN: Valor predictivo negativo. LR+: Razón de verosimilitud positiva. LR-: Razón de verosimilitud negativa. aROC: Área bajo la curva ROC.
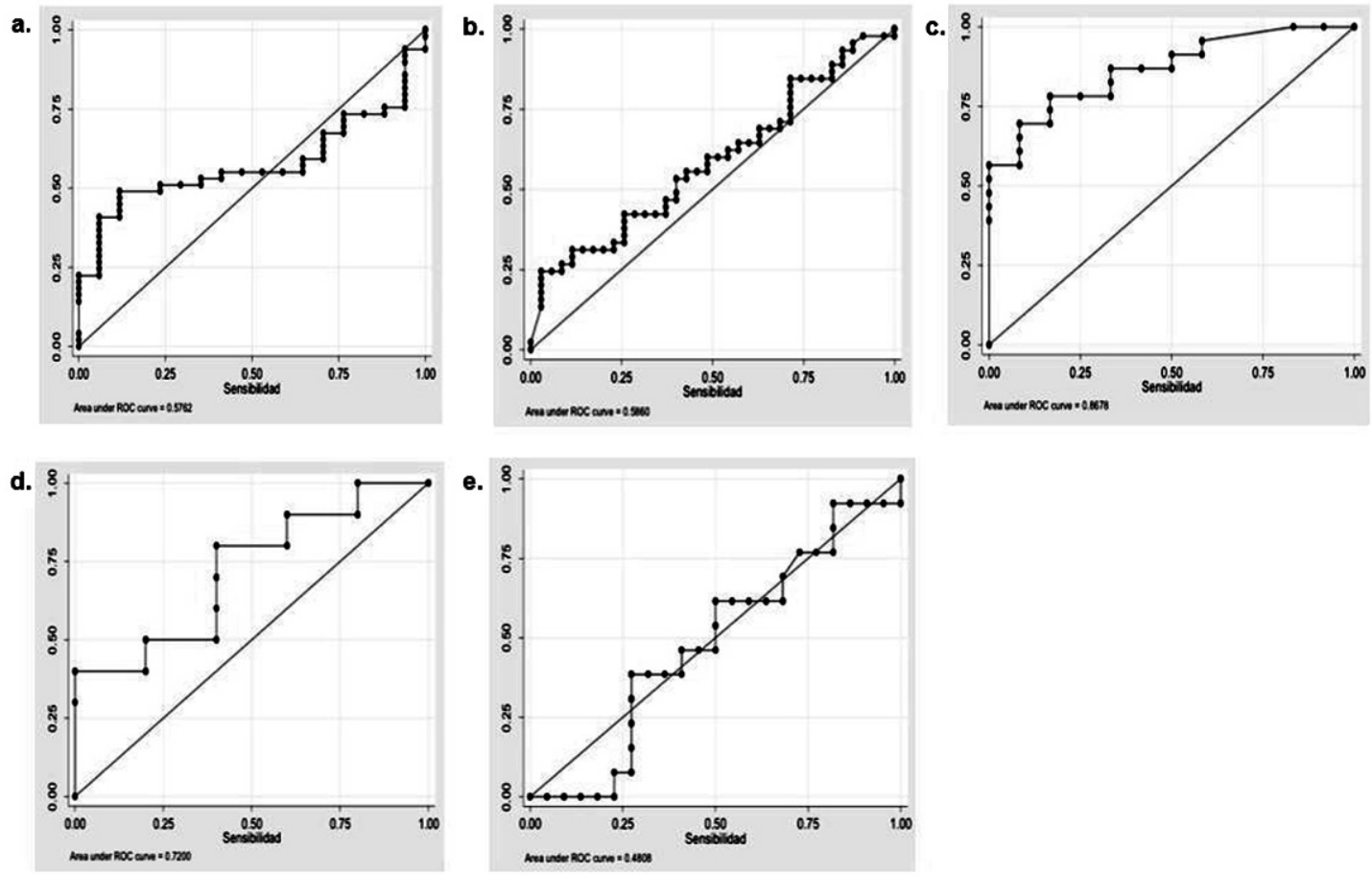

Figura 2. Áreas bajo las curvas ROC de los marcadores tumorales. a) ACE; b) AFP; c) CA 19-9; d) CA 125; e) PSA. 


\section{Discusión}

El diagnóstico oportuno del cáncer puede mejorar la supervivencia y calidad de vida de los pacientes; sin embargo, en un importante número de pacientes este se realiza en estadios avanzados. Para el caso de enfermedad metastásica el pronóstico es malo; aunque se describe cerca de $20 \%$ de estos pacientes con una probabilidad teórica de responder al manejo oncológico ${ }^{11}$, de tal forma que brindar un diagnóstico preciso y rápido, cobra vital importancia y disminuye la ansiedad generada por la incertidumbre de no lograr un diagnóstico oportuno.

Los MTSC son una herramienta sencilla, de bajo costo, accesible y de rápida ejecución; sin embargo, su indicación más estudiada corresponde al seguimiento de los pacientes con un diagnóstico establecido. Además, su sensibilidad y especificidad como ayuda diagnóstica es baja, razón por la cual las diferentes guías no los recomiendan en el abordaje diagnóstico.

Molina et al. ${ }^{11}$ evaluaron los MTSC, los cuales mostraron tener muy buen rendimiento diagnóstico en pacientes con enfermedad avanzada, condición que supuetamente los convertiría en una excelente ayuda en el abordaje de estos casos. Ese estudio propone diferentes puntos de corte según algunas características clínicas de los pacientes, tales como, la presencia de derrame pleural, ascitis o falla renal; sin embargo, en el presente informe no fue posible realizar análisis en los diferentes subgrupos clínicos por la escasa cantidad de pacientes reclutados para subgrupos durante el estudio. Con todo, las características clínicas de los pacientes aquí incluidos, en particular por la distribución de comorbilidades para cada grupo, hace que los criterios de selección en cada uno de ellos, correspondieran según la condición clínica (malignos o no), a estar o no asociada con enfermedad cirrótica y cambios en los niveles de MTSC.

El muestreo naturalístico o transveral, con el cual todos los pacientes con lesión maligna o no, tuvieron la misma posibilidad de ser seleccionados, hace posible estimar sin sesgos todos los indicadores de rendimiento diagnóstico. Así, todos menos uno de los MTSC evaluados presentan muy bajos índices de rendimiento diagnóstico, condición que no permite recomendar su uso para discriminar lesiones hepáticas malignas de las benignas. Solo el CA 19-9 tiene la capacidad para hacerlo; de hecho, es posible plantear un valor de corte propio para nuestra población con CA 19-9 de 17,6 U/ml para el cual se obtiene una capacidad discriminatoria para malignidad de $71 \%$. Este punto de corte es menor al descrito por Molina et al. ${ }^{11}$, quienes fijaron un punto de corte de $200 \mathrm{U} / \mathrm{ml}$ para este marcador; hallazgo este que exige estudios adicionales.

Es importante destacar que la poca o nula utilidad de los MTSC para el abordaje diagnóstico inicial de los pacientes con lesiones hepáticas potencialmente malignas hallada en el presente estudio no depende del tamaño de la muestra de pacientes estudiados, puesto que las curvas ROC cruzan la diagonal que representa la incapacidad de la prueba para discriminar los pacientes (como ocurre con ACE y PSA); o su comportamiento es casi paralelo a esta (como ocurre con AFP); de hecho, la capacidad discriminatoria del CA 19-9 se hizo evidente con muchos menos pacientes con relación a los evaluados para ACE, AFP o PSA. Por consiguiente, un incremento en el tamaño de muestra tan solo podría afinar los números para el Ca 125 en el cual solo se evaluaron 15 pacientes, pero con muy poca probabilidad para los otros tres marcadores.

Visto así, el enfoque diagnóstico de adultos con lesiones tumorales hepáticas sigue implicando una la alta correlación con la sintomatología que presenta cada paciente $y$, por consiguiente, es posible que el uso de MTSC deba reservarse para seguimiento de pacientes ya diagnosticados y, en casos muy selectos, como método diagnóstico. No es posible aún reemplazar el patrón de oro soportado por el diagnóstico histopatológico, especialmente en tumores epiteliales la negatividad del marcador tumoral no excluye el diagnóstico cáncer ${ }^{11}$.

Este estudio plantea la necesidad de racionalizar el recurso de los MTSC, dada su poca utilidad para enfocar el diagnóstico definitivo de los pacientes con sospecha de CPOMH. El uso de pruebas inapropiadas incrementa los resultados falsos positivos y negativos, y generan mayor confusión tanto para el médico como para el paciente.

\section{Referencias}

1. Lynn H, Hayes D. Uses and abuses of tumor markers in the diagnosis, monitoring, and treatment of primary 
and metastatic breast cancer. The oncologist 2006; 11: 541-52.

2. Ocaña E, Aceituno I. Utilidad clínica de los marcadores tumorales. Revista Médica de Jaén 2014; 4: 2-12.

3. Urban D, Catane R. Serum tumor markers in oncology. IMAJ 2009; 12: 103.

4. Sturgeon C. Practice guidelines for tumor marker use in the clinic. Clin Chem 2002; 48 (8): 1151-9.

5. Sturgeon CM, Duffy MJ, Stenman UH, Lilja H, Brünner $\mathrm{N}$, Chan DW, et al. National Academy of Clinical Biochemistry laboratory medicine practice guidelines for use of tumor markers in testicular, prostate, colorectal, breast, and ovarian cancers. Clin Chem 2008; 54 (12): e11-79.

6. Fleisher M, Dnistrian AM, Sturgeon CM, Lamerz R, Wittliff JL. Practice guidelines and recommendations for use of tumor marker in the clinic. In: Diamandis EP, Fritsche H, Schwartz MK, Chan DW, Editores. Tumor markers: physiology, pathobiology, technology and clinical applications. Chicago: AACC Press, 2002: 33-63.
7. Fletcher R. Carcinoembryonic antigen. Ann Intern Med 1986; 104: 66-73.

8. Locker G, Hamilton S, Harris J, Jessup J, Kemeny N, Macdonald J, et al. ASCO 2006 Update of recommendations for the use of tumor markers in gastrointestinal cancer. J Clin Oncol 2008; 24 (33): 5313-27.

9. European Society for Medical Oncology (ESMO). ESMO minimum clinical recommendations for diagnosis, treatment and follow up of ovarian cancer. Ann Oncol 2001; 12: 1205-7.

10. Bast RC Jr, Radvin P, Hayes DF, Bates S, Fritsche H Jr, Jessup JM, et al. 2000 update of recommendations for the use of tumor markers in breast and colorectal cancer: clinical practice guidelines of the American Society of Clinical Oncology. J Clin Oncol 2001; 19: 1865-78.

11. Molina R, Bosh X, Auge J, Filella X, Escudero J, Molina V. Utility of serum tumor markers as an aid in the differential diagnosis of patients with clinical suspicion of cancer and in patients with cancer of unknown primary site. Tumor Biol 2012; 33: 463-74. 